\title{
Random effects meta-analysis of COVID-19/S. aureus partnership in co-infection
}

\section{Random-Effekt-Meta-Analyse von COVID-19/S. aureus Partnerschaft bei Co-Infektionen}

\begin{abstract}
Aim: To assess the hypothesis that coinfection with SARS-CoV-2 and S. aureus exacerbates morbidity and mortality among patients, the study aims to report the pooled burden of $S$. aureus co-infections in patients hospitalized with COVID-19.

Methods: We searched electronic databases and the bibliographies of pertinent papers for articles. We considered studies in which the core result was the number of patients with bacterial (S. aureus) co-infection. We performed random effects meta-analysis (REM) because the studies included were sampled from a universe of different populations and high heterogeneity was anticipated. Using the Cochran's Q statistic, the observed dispersion (heterogeneity) among effect sizes was assessed. The percentage of total variability in the estimates of the effect size was calculated with the $\mathrm{I}^{2}$ index. To check for publication bias, the Egger weighted regression, Begg rank correlation and meta-funnel plot were used. We conducted meta-regression analysis to evaluate the variability between our outcomes and the covariates using computational options such as "methods of moments" and then "maximum likelihood" ratio. Results: We included 18 studies and retrieved data for 63,370 patients hospitalized with influenza-like illness, of which about 14,369 (22.67\%) tested positive for COVID-19 by rRT-PCR. Of this number, 8,249 (57.4\%) patient samples were analyzed. Bacterial, fungal and viral agents were detected in 3,038 (36.8\%); S. aureus in 1,192 (39.2\%). Five studies reported MRSA co-infection. Study quality ranged from 6 to 9 (median 7.1) on a JBI scale. From the meta-analysis, $33.1 \%$ patients were found to be coinfected $\left(95 \%, \mathrm{Cl} 18.0\right.$ to $52.6 \%, \mathrm{Q}=3473$ : $\mathrm{df}=17, \mathrm{I}^{2}=99.48 \%$, $p=0.00)$. The rate of S. aureus /COVID-19 co-infection was $25.6 \%(95 \%$ $\mathrm{Cl}$ : 15.6 to $\left.39.0, \mathrm{Q}=783.4, \mathrm{df}=17, \mathrm{I}^{2}=97.702 \%, \mathrm{p}=0.003\right)$. . The proportion of COVID-19/S. aureus co-infected patients with MRSA was $53.9 \%$ (95\% $\mathrm{Cl}, 24.5$ to 80.9, $\mathrm{n}=66,5$ studies, $\mathrm{Q}=29.32, \mathrm{df}=4, \mathrm{I}^{2}=86.369 \%$, $\mathrm{p}=0.000$ ). With the multivariate meta-regression model, study type $(p=0.029)$, quality $(p=0.000)$ and country $(p=0.000)$ were significantly associated with heterogeneity.

Conclusions: The pooled rates of S. aureus among COVID-19 patients documented in this study support the concern of clinicians about the presence of $S$. aureus in co-infections. Improved antibiotic stewardship can be accomplished through rapid diagnosis by longitudinal sampling of patients.
\end{abstract}

Keywords: COVID-19, S. aureus, co-infection, meta-analysis, meta-regression

\section{Zusammenfassung}

Ziel: Zur Überprüfung der Hypothese, dass die Koinfektion von SARSCoV-2 mit S. aureus die Morbidität und Mortalität verschlimmert, sollte das Outcome bei Koninfektionen bei mit COVID-19 hospitalisierten Patienten analysiert werden.

\section{Suleiman Shuaibu \\ Adeiza $^{1}$ \\ Abdulmalik Bello \\ Shuaibu $^{2}$ \\ Gazali Mohammed \\ Shuaibu $^{3}$}

1 Department of Pharmaceutical Microbiology, Faculty of Pharmaceutical sciences, Ahmadu Bello University, Zaria, Kaduna, Nigeria

2 Department of Veterinary Microbiology, Faculty of Veterinary medicine, Usmanu Danfodiyo University, Sokoto, Nigeria

3 Department of Microbiology, Faculty of sciences, Usmanu Danfodiyo University, Sokoto, Nigeria 
Methode: Bei der Recherche in elektronischen Datenbanken und Bibliographien wurden alle Studien mit Co-Infektion von COVID-19 und S. aureus berücksichtigt. Wir führten eine random effects meta-analysis (REM) durch, da die eingeschlossenen Studien aus verschiedenen Populationen ausgewählt wurden und eine hohe Heterogenität erwartet wurde. Mit Hilfe der Cochran's Q-Statistik wurde die beobachtete Streuung (Heterogenität) zwischen den Effektgrößen bewertet. Der Prozentsatz der Gesamtvariabilität bei den Schätzungen der Effektgröße wurde mit dem I'-Index berechnet. Zur Überprüfung des Publikationsbias wurden die Egger-gewichtete Regression, die Begg-Rang-Korrelation und das Meta-Tunnel-Plot verwendet. Wir führten eine Meta-Regressionsanalyse durch, um die Variabilität zwischen unseren Ergebnissen und den Kovariaten unter Verwendung von Berechnungsoptionen wie „Momentmethoden“ und dann „Maximum-Likelihood“-Verhältnis zu bewerten.

Ergebnisse: Es wurden achtzehn Studien mit Daten für 63.370 Patienten eingeschlossen, die mit grippeähnlicher Erkrankung ins Krankenhaus eingewiesen wurden. Davon wurden 14.369 (22,7\%) mittels rRT-PCR positiv auf COVID-19 getestet. Hiervon wurden 8.249 (57,4\%) Patientenproben analysiert. Bakterielle, pilzliche und virale Erreger wurden bei 3.038 (36,8\%), S. aureus bei 1.192 (39,2\%) nachgewiesen. Fünf Studien berichteten über eine MRSA-Koinfektion. Die Studienqualität reichte von 6 bis 9 (Median 7,1) auf der JBI-Skala. Aus der Meta-Analyse ging hervor, dass 33,1\% der Patienten koinfiziert waren (95\%, Cl 18,0 bis 52,6\%, Q=3473: $\left.d f=17, I^{2}=99-48 \%, p=0,00\right)$. Die Rate der Koinfektion von S. aureus/COVID-19 betrug 25,6\% (95\% KI: 15,6 bis 39,0, $\left.\mathrm{Q}=783,4, \mathrm{df}=17, \mathrm{I}^{2}=97,702 \%, \mathrm{p}=0,003\right)$. Der Anteil von COVID-19/S. aureus ko-infizierten Patienten mit MRSA betrug 53,9\% (95\% KI, 24,5 bis 80,9, $n=66,5$ Studien, $\left.Q=29,32, d f=4, I^{2}=86,369 \%, p=0,000\right)$. Beim multivariaten Meta-Regressionsmodell waren Studientyp $(p=0,029)$, Qualität $(p=0,000)$ und Land $(p=0,000)$ signifikant mit Heterogenität assoziiert.

Schlussfolgerung: Die in dieser Studie dokumentierten gepoolten Raten von S. aureus Infektion bei COVID-19-Patienten unterstützen die Besorgnis der Kliniker hinsichtlich des Ausmaßes der Bakterien bei Co-Infektionen. Eine verbesserte Antibiotika-Stewardship kann durch eine schnelle Diagnose mittels Längsschnitt-Stichproben von Patienten erreicht werden.

Schlüsselwörter: COVID-19, S. aureus, Co-Infektion, Meta-Analyse, Meta-Regression

\section{Introduction}

The morbidity and mortality rate associated with COVID19 is not unrelated to co-infections with bacterial pathogens [1]. Respiratory and blood culture studies of hospitalized patients with severe acute respiratory coronavirus 2 (SARS-CoV-2) have shown that bacterial infections rather than the direct effects of the virus have resulted in a number of recorded fatalities [2].

Staphylococcus aureus (S. aureus) is persistently and asymptomatically present in the nares of $20 \%$ of the human population [3], [4]. In response to physiological changes during infection, bacterial factors involved in the shift of $S$. aureus from commensalism to pathogenesis is poorly understood [5]. The bacteria have been associated with secondary staphylococcal pneumonia following COVID-19 infection [6], [7]. However, the overlap of symptoms makes the identification of co-infected patients and the co-infecting pathogens laborious [8].

Although previous studies investigated the frequency of selected bacterial species in COVID-19 cases [9] and the overall extent of co-infection [10], the pooled rate of $S$. aureus among hospitalized COVID-19 patients is largely undocumented. This study aims to address this issue by conducting a meta-analysis to determine the burden of S. aureus co-infections in patients hospitalized with COVID-19. Knowledge about specific etiological agents may reduce the strain on the resources of healthcare systems worldwide and lead to more appropriate treatment and medication, as well as shorter hospitalization. 


\section{Methods}

\section{Search strategy, selection criteria and data extraction}

We examined databases for studies that reported data on S. aureus and MRSA co-infections in patients with COVID-19 infection. Studies with fewer than 10 participants and case studies were excluded. Searches were performed in Pub Med, Google Scholar, Web of Science and SCOPUS from $1^{\text {st }}$ of January 2020 up to $20^{\text {th }}$ of October 2020. The search terms included: 'COVID-19 and MRSA', 'bacterial infection and MRSA', 'S. aureus and COVID-19', 'SARS-CoV-2 and MRSA or S. aureus' 'bacterial pathogens and COVID-19'. These were combined with search terms such as 'hospital', 'healthcare', 'communityacquired', ‘hospital-acquired', 'bacteremia', 'pneumonia', 'secondary infections', 'supra-infection', 'co-infection'. The bibliographies of identifed articles were also searched. The abstracts and full texts of identified studies were screened for eligibility by two reviewers (SSA and ABS). The quality of studies was evaluated using the Joanna Briggs Institute Checklist for Studies Reporting Prevalence Data [11]. Discrepancies in evaluation were settled by consultation with a mediator (SMG). PRISMA (preferred reporting items for systematic reviews and meta-analyses) protocols were used for this analysis [12]. The data gathered from the included studies comprised author's name, country of study, type of study, setting, culture type, and number of patients with: influenza-like illness (ILI), COVID-19 positive results, co-infections, S. aureus co-infections or MRSA co-infections.

\section{Data analysis and assessment of bias}

Random effects meta-analysis (REM) was performed because the studies included were sampled from a universe of different populations and high heterogeneity was anticipated. Comprehensive Meta-Analysis ${ }^{\circledR}$ software, version 3.3070 (Bio-stat, Englewood, NJ, USA), was used to perform the analysis. Using the Cochran's Q statistic, the observed dispersion (heterogeneity) among effect sizes was assessed. The percentage of total variability in the estimates of the effect size was calculated with the $\mathrm{I}^{2}$ index. To check for publication bias, Egger weighted regression and Begg rank correlation methods with a metafunnel plot were used. A p-value $<0.05$ was presumed to reflect a statistically significant publication bias. In order to structure the search results and document relevant studies, Zotero desktop ${ }^{\circledR}$ (version 5.0.92) reference software was employed. Sensitivity analyses were carried out to gauge the impact of each study (by omission) on the pooled rates [13].

Meta-regression analysis was conducted to evaluate the variability between our outcomes and the covariates (study type, study quality, setting and country). Covariates were first tested individually in a univariate analysis and then simultaneously in a multiple meta-regression model through the computational options "methods of moments" followed by the "maximum likelihood" ratio. The $\mathrm{R}^{2}$ analog was used to compute the total variance of all studies about the grand mean effects. Outlier diagnostics were performed using Cook's distances, covariance ratios, heterogeneity test statistics and weights (Attachment Fig. S4).

\section{Results}

Our search yielded 207 titles after removing duplicates, of which 148 were removed during the initial screening. At this point, the abstracts of the outstanding 59 studies were reviewed, and 41 studies were discarded because they did not meet the inclusion criteria, leaving 18 studies included in the study (Figure 1). The study quality ranged from 6 to 9 (median 7.1) on the Joanna Briggs Institute scale. All included studies reported on S. aureus/COVID19 co-infection amongst hospitalized patients. Table 1 provides data on 63,370 patients from the included studies. Five studies were from the United States (27.8\%), 2 each from the United Kingdom, China, and Italy (33.3\%), 1 each from France, Egypt, Saudi Arabia, Netherlands, Spain, Iran and Russia (38.9\%). We retrieved data for the 63,370 patients hospitalized with influenza-like illness, about 14,369 (22.67\%) of which tested positive for COVID-19 by rRT-PCR. Of this number, 8,249 (57.4\%) of patient samples (respiratory and blood) were analyzed for co-infecting pathogens. Bacterial, fungal and viral agents were detected in 3,038 (36.8\%) patients, and S. aureus in 1,192 (39.2\%) patients. Only five studies reported MRSA co-infection in 66 patients.

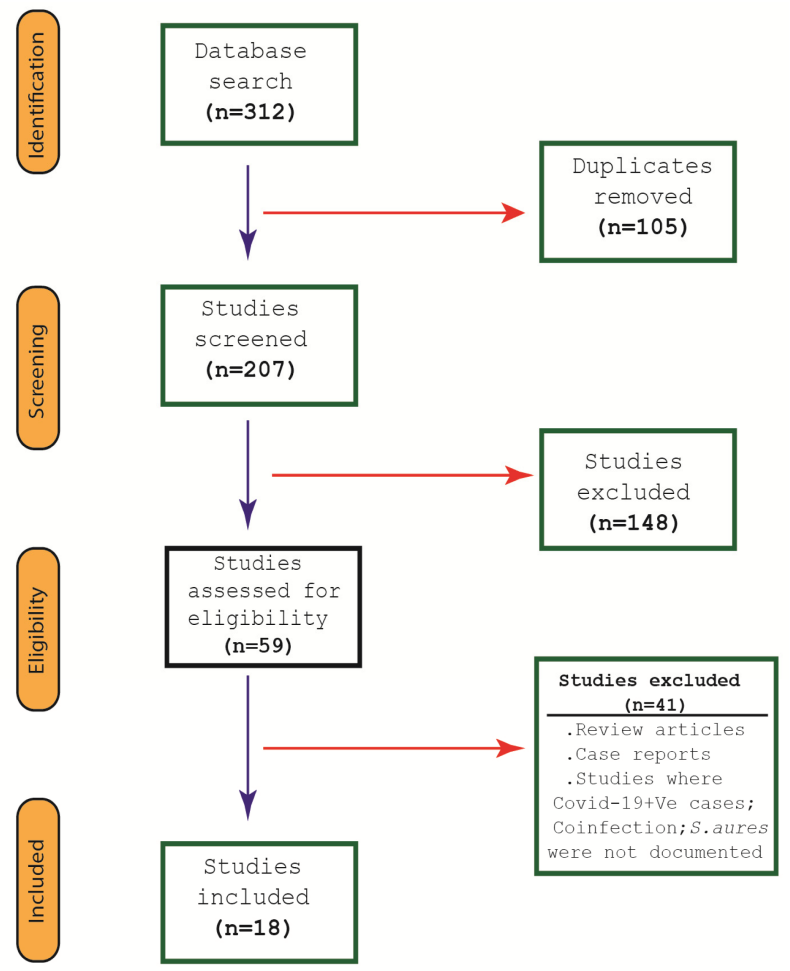

Figure 1: Flow diagram of article selection process 
Table 1: Study characteristics

\begin{tabular}{|c|c|c|c|c|c|c|c|c|c|c|c|c|c|c|c|c|c|c|}
\hline 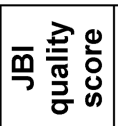 & & & & & & & & & $\begin{array}{lll}0 & 6\end{array}$ & 0 & & & & & 0 & & \begin{tabular}{l|l}
$\infty$ & $\wedge$
\end{tabular} & \\
\hline \multirow{6}{*}{ 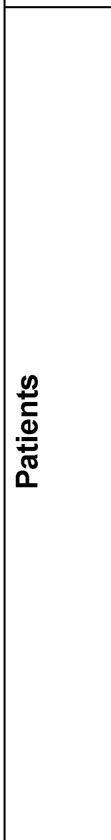 } & 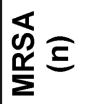 & $\mathbb{z}$ & $\mathbb{Z}$ & $m$ & $\mathbb{z}$ & $\mathbb{z}$ & $\mathbb{z}$ & $r$ & $\mathbb{Z} \mathbf{b}$ & ' & প্ল & 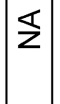 & $\hat{N}$ & \begin{tabular}{|}
$\mathbb{z}$ \\
$\mathbf{z}$
\end{tabular} & $\mathbb{z}$ & $\$ 2$ & $\begin{array}{l}\mathbf{z} \\
\mathbf{z}\end{array}$ & $\mathscr{\bullet}$ \\
\hline & 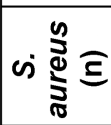 & $\nabla$ & $\bar{N}$ & ம & : & \begin{tabular}{|l|}
$\infty$ \\
-
\end{tabular} & \begin{tabular}{l|c}
$\infty$ & \multicolumn{1}{c}{}
\end{tabular} & \begin{tabular}{c|c}
$N$ & $c$
\end{tabular} & \begin{tabular}{l|l}
$N$ & 15
\end{tabular} & م| & $\hat{f}$ & $N$ & $\underset{工}{\mathbb{N}}$ & \begin{tabular}{l|l}
$*$ \\
$\nabla$
\end{tabular} & 0 & 이요 & 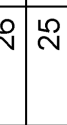 & 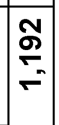 \\
\hline & ن & m & $\stackrel{\mathfrak{N}}{\mathrm{N}}$ & $\stackrel{\infty}{+}$ & $\begin{array}{l}\mathbb{2} \\
\infty \\
0\end{array}$ & $\bar{\sigma}$ & $\begin{array}{l}\mathscr{0} \\
0 \\
0\end{array}$ & \& & ه & $\begin{array}{lll}\circ & 0\end{array}$ & న & 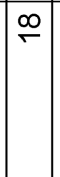 & $\begin{array}{l}\mathcal{V} \\
\tilde{v}\end{array}$ & ষ & $\stackrel{f}{q}$ & 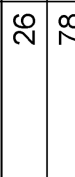 & 巳 & 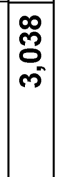 \\
\hline & 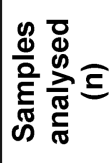 & $\stackrel{ }{\rightleftharpoons}$ & 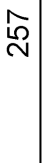 & $\stackrel{\mathscr{D}}{\sim}$ & \begin{tabular}{l}
$\widetilde{\infty}$ \\
0 \\
\hdashline \\
-
\end{tabular} & $\hat{f}$ & 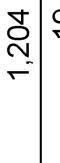 & 믹 & 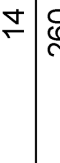 & 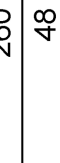 & $\stackrel{N}{\mathfrak{S}}$ & 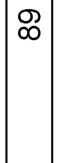 & $\begin{array}{l}\bar{N} \\
\underset{\sigma}{*}\end{array}$ & 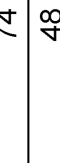 & $\stackrel{\infty}{\wedge}$ & $\delta \stackrel{\infty}{\alpha}$ & $\begin{array}{l}0 \\
0\end{array}$ & 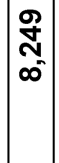 \\
\hline & 官 & $\mid \begin{array}{l}\infty \\
\infty \\
\infty\end{array}$ & స્⿱ & $\stackrel{\mathbb{D}}{\stackrel{\infty}{\sim}}$ & $\begin{array}{c}\stackrel{m}{\sigma} \\
\stackrel{-}{-}\end{array}$ & $\stackrel{f}{\leftarrow}$ & $\begin{array}{l}\text { Dે } \\
\text { - } \\
-\end{array}$ & อ & 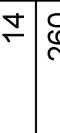 & on & 중 & $\infty$ & $\begin{array}{l}\bar{N} \\
\underset{\sim}{\sim}\end{array}$ & $\begin{array}{l}\mathbb{b}^{\infty} \\
\stackrel{\infty}{\infty}\end{array}$ & $\stackrel{\infty}{\wedge}$ & 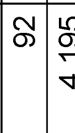 & 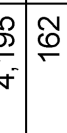 & 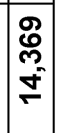 \\
\hline & $\Xi \widehat{\Xi}$ & \begin{tabular}{l}
$\tilde{\delta}$ \\
\hdashline \\
-
\end{tabular} & సి & $\stackrel{\tilde{\sigma}}{\dot{\sigma}}$ & $\begin{array}{c}\infty \\
\stackrel{f}{0} \\
m \\
m\end{array}$ & $\mid$ & $\begin{array}{l}\underset{\text { D̦ }}{-} \\
-\end{array}$ & - & 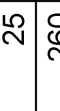 & 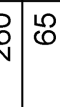 & 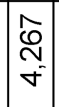 & $\infty$ & $\underset{\underset{\sim}{\grave{*}}}{\bar{\sim}}$ & 必 & $\stackrel{\infty}{\wedge}$ & 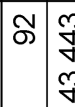 & 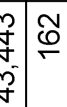 & $\begin{array}{c}0 \\
0 \\
0 \\
0 \\
0 \\
0\end{array}$ \\
\hline 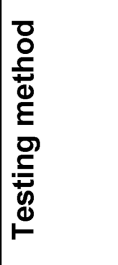 & & 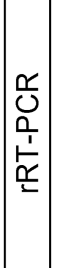 & $\begin{array}{l}\frac{r}{0} \\
0 \\
\frac{1}{1} \\
\underline{\underline{x}} \\
\mathbf{x}\end{array}$ & $\begin{array}{l}\frac{x}{0} \\
0 \\
1 \\
\underline{\underline{x}} \\
\underline{\underline{x}}\end{array}$ & $\begin{array}{l}\frac{\underline{c}}{0} \\
\frac{0}{1} \\
\underline{\underline{\alpha}} \\
\underline{\underline{x}}\end{array}$ & 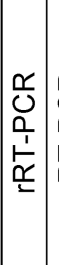 & 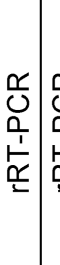 & 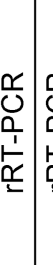 & 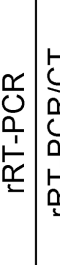 & 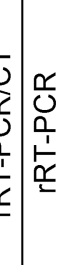 & 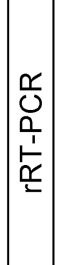 & 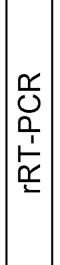 & 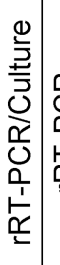 & 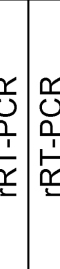 & $\begin{array}{l} \\
\underline{\underline{u}} \\
0 \\
\frac{1}{\alpha} \\
\underline{\underline{x}}\end{array}$ & 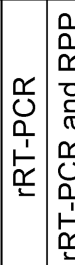 & 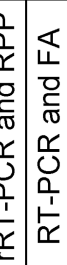 & \\
\hline 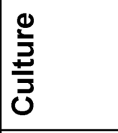 & & U & $\begin{array}{l}0 \\
\propto\end{array}$ & $\begin{array}{l}0 \\
\mathbb{x}\end{array}$ & O্ & $\begin{array}{l}0 \\
\mathbb{x}\end{array} \mid$ & 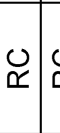 & Ur| & $\begin{array}{l}0 \\
\mathbb{\Psi} \\
\end{array}$ & $\begin{array}{l}0 \\
\mid\end{array}$ & $\begin{array}{l}0 \\
\mathbb{x}\end{array}$ & \begin{tabular}{|l|}
0 \\
$\ltimes$
\end{tabular} & O্ৰ & $\underset{r}{v}$ & 邑 & O্口 & $\begin{array}{l}0 \\
\underline{x}\end{array}$ & \\
\hline 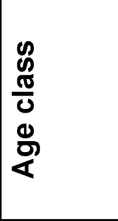 & & $\mid \frac{2}{\frac{2}{3}}$ & $\frac{\frac{0}{3}}{\frac{3}{4}}$ & & $\frac{\frac{0}{5}}{\frac{3}{\alpha}}$ & 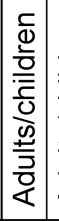 & 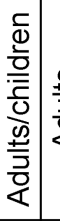 & $\frac{9}{\frac{2}{3}} \frac{5}{4}$ & 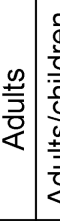 & 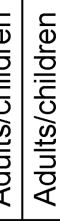 & $\frac{\frac{2}{3}}{\frac{3}{4}}$ & 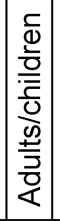 & & 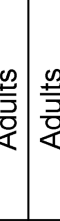 & $\frac{\frac{0}{5}}{\frac{2}{0}}$ & 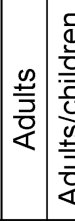 & 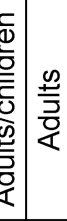 & \\
\hline $\begin{array}{l}\text { वे } \\
\text { क्ष }\end{array}$ & & $\begin{array}{l}\infty \\
\mathbb{x}\end{array} \mid$ & $\infty$ & $\infty$ & $\infty$ & $\begin{array}{l}\infty \\
\Sigma\end{array}$ & 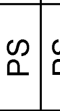 & $\infty$ & $\begin{array}{c}\infty \\
0 \\
0\end{array}$ & $\begin{array}{lll}2 & \infty\end{array}$ & $\mid \begin{array}{c}\infty \\
\mathbb{x}\end{array}$ & $\begin{array}{l}\infty \\
0\end{array}$ & क & is 2 & $\begin{array}{l}\mathscr{D} \\
\mathbb{L}\end{array}$ & \begin{tabular}{lll}
$\mathscr{L}$ & $\mathscr{\sim}$ \\
\hdashline
\end{tabular} & $\stackrel{\infty}{2}$ & \\
\hline 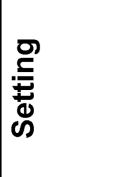 & & 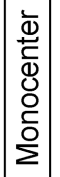 & 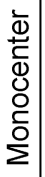 & 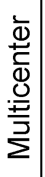 & 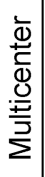 & 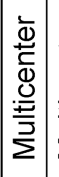 & 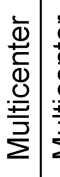 & 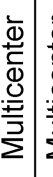 & 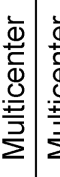 & 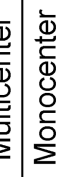 & 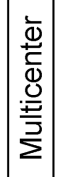 & 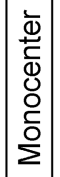 & 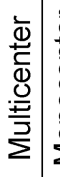 & 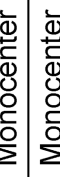 & 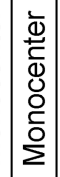 & 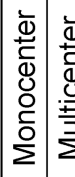 & 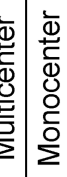 & \\
\hline \begin{tabular}{l} 
D̂ \\
\multirow{5}{*}{} \\
0 \\
0
\end{tabular} & & 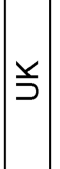 & 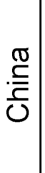 & 审 & $\begin{array}{l}\overleftarrow{\Phi} \\
\stackrel{D}{د}\end{array}$ & 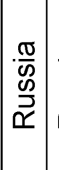 & 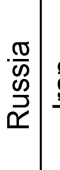 & 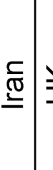 & כ઼ & 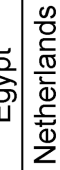 & 芯 & 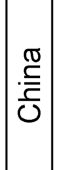 & ङ & 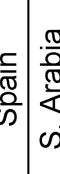 & 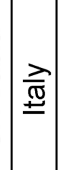 & 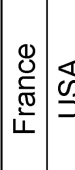 & 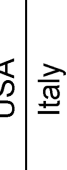 & \\
\hline 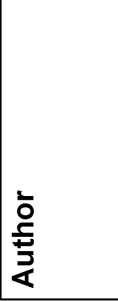 & & 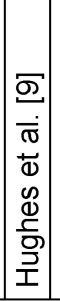 & 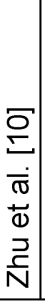 & 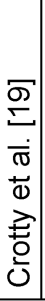 & 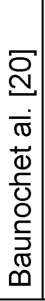 & 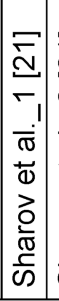 & 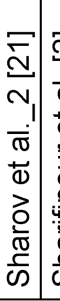 & 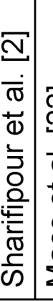 & 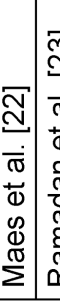 & 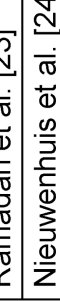 & 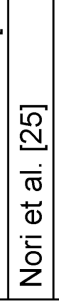 & 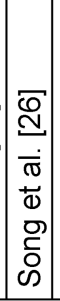 & 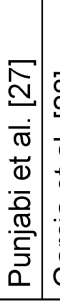 & 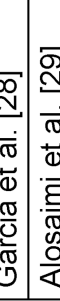 & 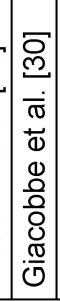 & 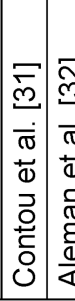 & 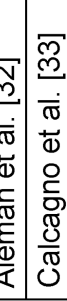 & 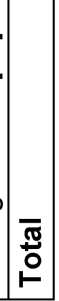 \\
\hline
\end{tabular}




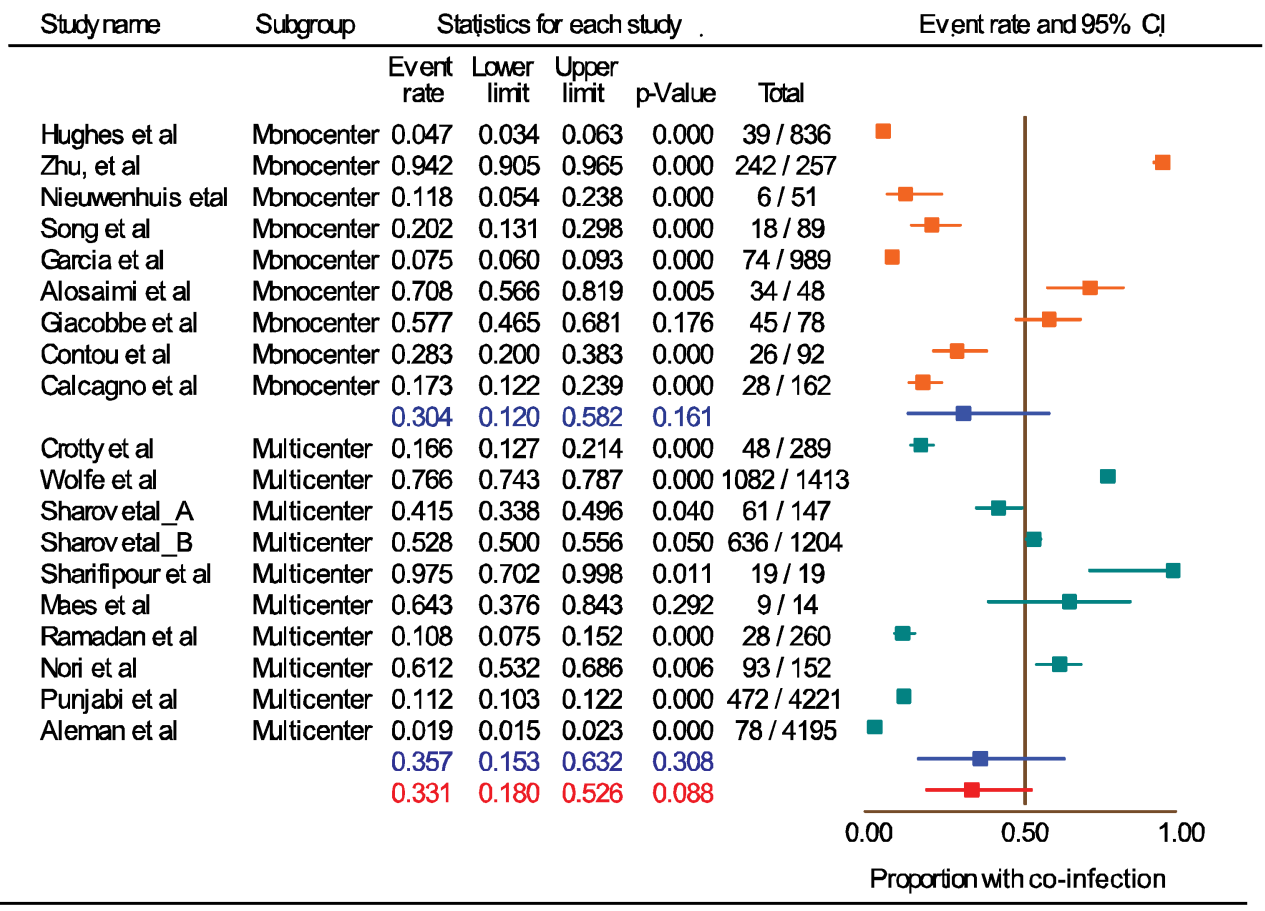

\begin{tabular}{|c|c|c|c|c|c|c|}
\hline \multirow[t]{2}{*}{ Study name } & \multirow[t]{2}{*}{ Subgroup } & \multicolumn{4}{|c|}{ Statistics with study removed } & \multirow{2}{*}{$\begin{array}{l}\text { Event rate }(95 \% \mathrm{Cl}) \\
\text { with study removed }\end{array}$} \\
\hline & & Point & $\begin{array}{l}\text { Lower } \\
\text { limit }\end{array}$ & $\begin{array}{c}\text { Upper } \\
\text { limit }\end{array}$ & p-Value & \\
\hline Hughes et al & Mbnocenter & 0.360 & 0.200 & 0.559 & 0.165 & \\
\hline Zhu, et al & Mbnocenter & 0.288 & 0.154 & 0.473 & 0.026 & $\rightarrow$ \\
\hline Crotty et a & Multicenter & 0.342 & 0.185 & 0.545 & 0.124 & $\rightarrow$ \\
\hline Wolfee a & Multicenter & 0.303 & 0.174 & 0.473 & 0.024 & $\rightarrow$ \\
\hline Sharov eta $A$ & Multicenter & 0.326 & 0.174 & 0.526 & 0.087 & $\rightarrow$ \\
\hline Sharov eta_B & Miticenter & 0.322 & 0.164 & 0.536 & 0.100 & $\rightarrow$ \\
\hline Sharifipour et al & Multicenter & 0.299 & 0.160 & 0.488 & 0.038 & $\rightarrow$ \\
\hline Mes et al & Multicenter & 0.316 & 0.169 & 0.510 & 0.063 & $\rightarrow$ \\
\hline Ramadan et al & Multicenter & 0.349 & 0.190 & 0.549 & 0.137 & $\rightarrow$ \\
\hline Nieumerhuis eta & Monocenter & 0.346 & 0.190 & 0.545 & 0.128 & $\rightarrow$ \\
\hline Nori et al & Multicenter & 0.316 & 0.168 & 0.513 & 0.066 & $\rightarrow$ \\
\hline Song et al & Mbnocenter & 0.339 & 0.184 & 0.539 & 0.111 & $\rightarrow$ \\
\hline Punjabi et al & Multicenter & 0.349 & 0.186 & 0.557 & 0.153 & $\rightarrow$ \\
\hline Gercia et al & Mbnocenter & 0.354 & 0.194 & 0.555 & 0.152 & $\rightarrow$ \\
\hline Alosaimi et al & Mbnocenter & 0.311 & 0.166 & 0.505 & 0.056 & $\rightarrow-$ \\
\hline Gacobbe et al & Monocenter & 0.318 & 0.170 & 0.514 & 0.068 & $\rightarrow-$ \\
\hline Contou et al & Mbnocenter & 0.333 & 0.180 & 0.533 & 0.100 & $\rightarrow-$ \\
\hline Aleman et al & Multicenter & 0.371 & 0.217 & 0.556 & 0.168 & $\rightarrow$ \\
\hline \multirow[t]{3}{*}{ Calcagno et al } & Mbnocenter & 0.342 & 0.185 & 0.542 & 0.119 & $\rightarrow-$ \\
\hline & & 0.330 & 0.182 & 0.522 & 0.081 & $\rightarrow$ \\
\hline & & & & & & $\begin{array}{lll}0.00 & 0.50 & 1.0 \\
\text { Propartion with Co-infection }\end{array}$ \\
\hline
\end{tabular}

Figure 2: Frequency of co-infection in hospitalized COVID-19 patients (top); sensitivity analysis of the meta-analysis (bottom)

The Forest plots (Figure 2, Figure 3, and Figure 4) show the pooled rates of co-infections, S. aureus/COVID- 19 co-infection and frequency of MRSA among co-infected patients. Subgroup analysis was performed to evaluate whether the pooled effects differed for mono-center and multicenter studies. The red and blue summary symbols represent the overall and subgroup effect outcome of the analysis, respectively, at a confidence interval of $95 \%$. The squares signify the point estimates of each study and the square's size denotes the weight given in the metaanalysis. From pooled analysis of 18 studies, 33.1\% of patients reported co-infection (95\%, Cl 18.0 to $52.6 \%$, 


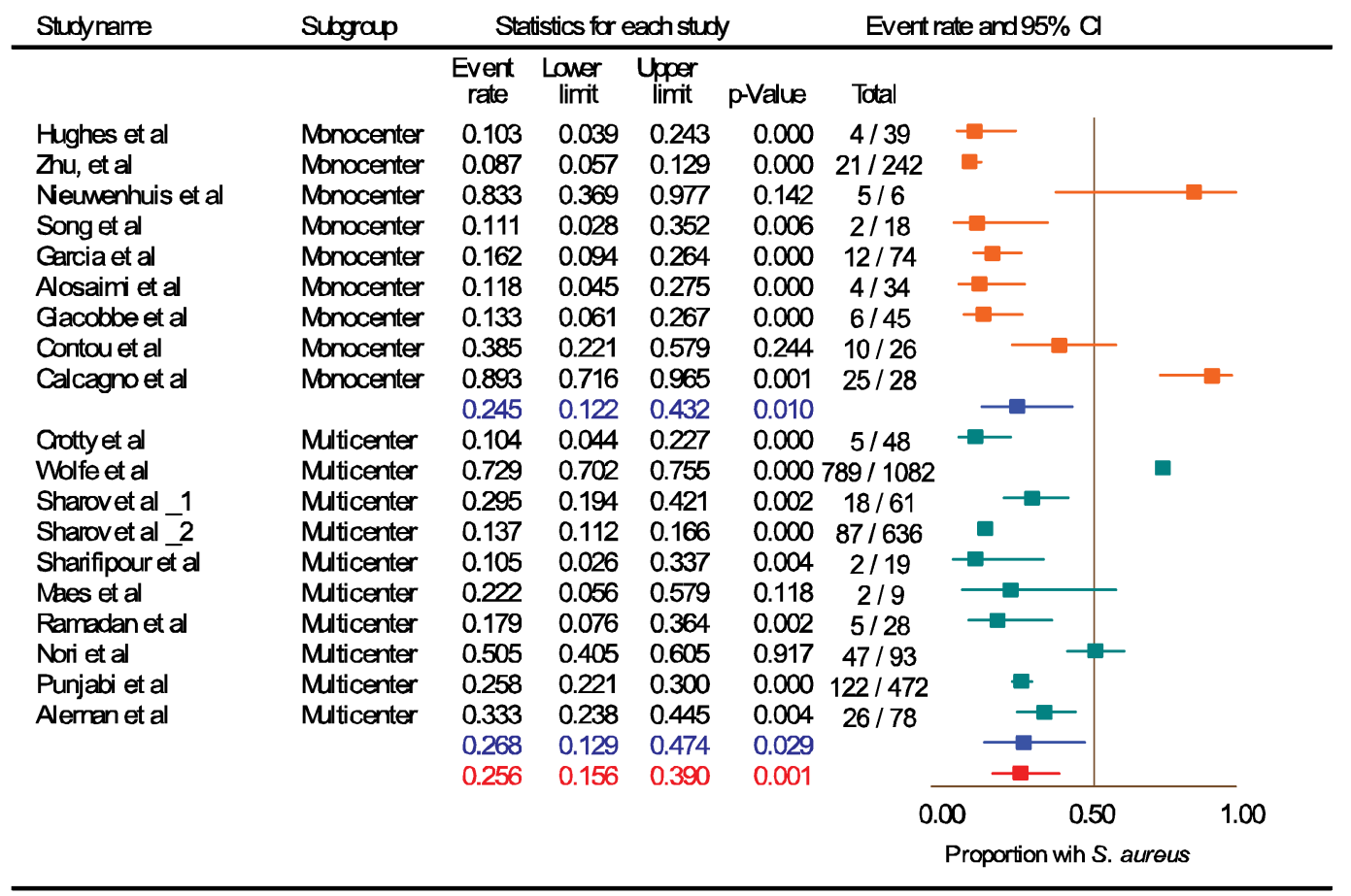

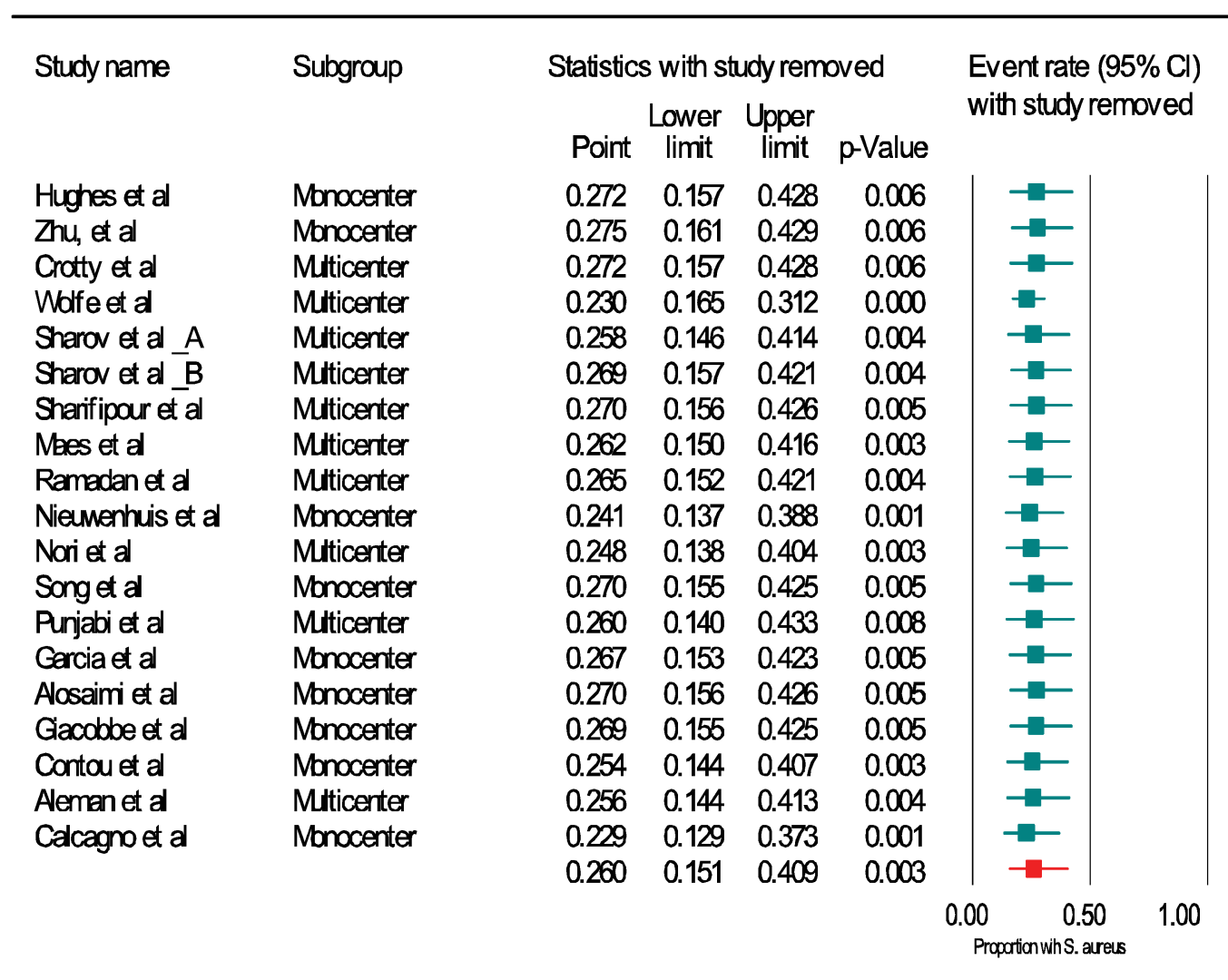

Figure 3: Frequency of $S$.aureus among hospitalized COVID-19 patients with co-infection (Top); sensitivity analysis of the meta-analysis (bottom)

$\left.\mathrm{Q}=3473: \mathrm{df}=17, \mathrm{l}^{2}=99.48 \%, \mathrm{p}=0.00\right)$ : mono-center, $30.4 \%$ (95\% Cl, 12.0 to 58.0\%); multicenter, 35.7\% (95\% Cl, 15.3 to $63.2 \%)$. Additionally, the pooled rate of S. aureus/COVID-19 co-infection among patients was $25.6 \%$ (95\% Cl: 15.6 to $39.0, Q=783.4, d f=17, I^{2}=97.702 \%$, $\mathrm{p}=0.003)$ : mono-center, $24.5 \%(95 \% \mathrm{Cl}, 12.2$ to $43.2 \%$, $\mathrm{p}=0.010)$; multicenter, $26.8 \%(95 \% \mathrm{Cl}, 12.9$ to $47.4 \%$, $\mathrm{p}=0.029)$. The overall pooled proportion of hospitalized COVID-19/S. aureus co-infected patients with MRSA was 53.9\% (95\% Cl, 24.5 to 80.9, $n=66,5$ studies, $Q=29.32$, 


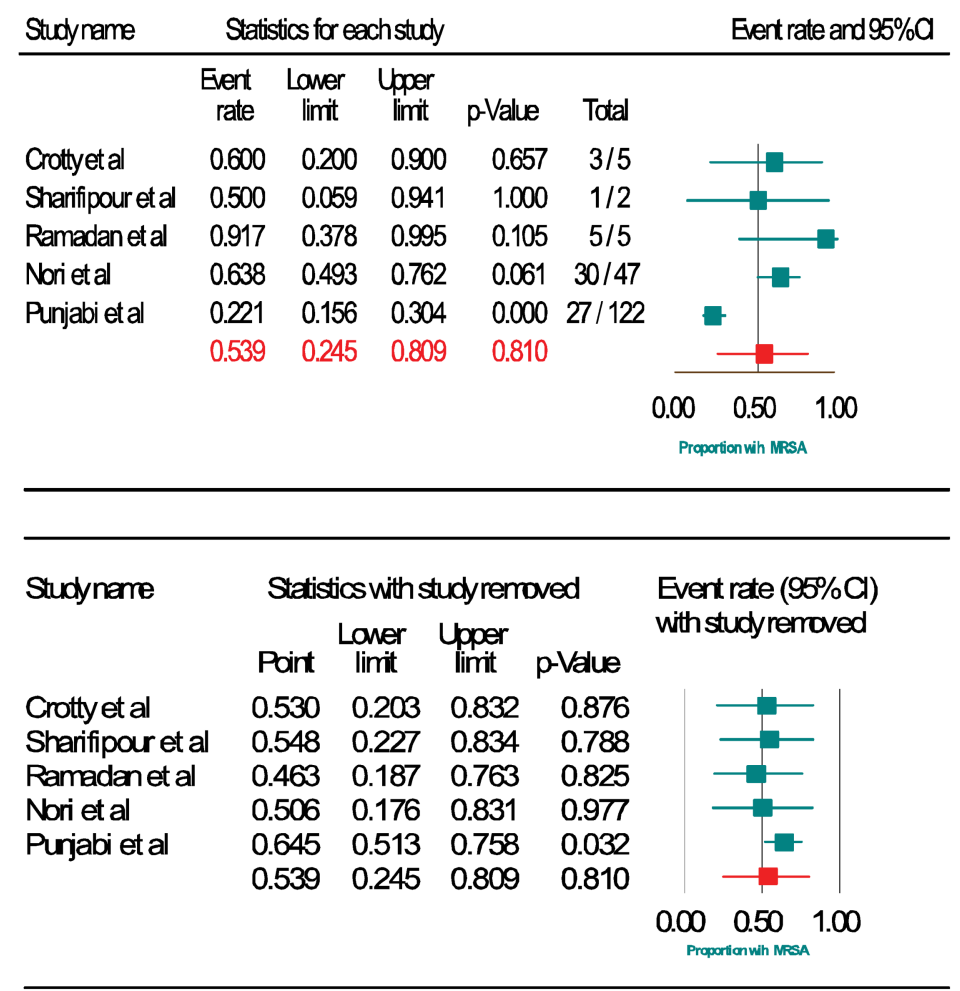

Figure 4: Frequency of MRSA among hospitalized COVID-19 patients with S. aureus co-infection (top); sensitivity analysis of the meta-analysis (bottom)

$\left.\mathrm{df}=4, \mathrm{I}^{2}=86.369 \%, \mathrm{p}=0.000\right)$. The sensitivity analysis did not significantly affect the overall proportion of our results by excluding one study, nor did it affect the heterogeneity. Some indication of publication bias among the analysed studies is shown by the asymmetric distribution of the studies in the funnel plots (Attachement Fig. S1, Fig. S2, and Fig. S3), highlighting the statistical heterogeneity observed. Egger's and Begg's tests (Attachement Tab. S1) did not demonstrate statistical significance for bias in any of the analyses ( $p>0.05)$. None of our covariates were statistically significant using the "methods of moment's" computation method. In the multivariate meta-regression model (maximum likelihood method), study type $(p=0.029)$, study quality $(p=0.000)$ and country of study $(p=0.000)$ were significantly associated with heterogeneity of results (Attachement Fig. S4). Of all the moderator variables, only study settings was not statistically significant (0.123). Overall, at a Q-value of 53.79 , with $\mathrm{df}=13$ and $p=0.000$, the covariates were associated with our observed effect. The variance of true effect sizes at any point on the regression line $\left(\mathrm{T}^{2}\right)$ was $0.2048, \mathrm{p} \leq 0.05$. Only $83 \%\left(R^{2}=0.83\right)$ of the variance in true effects can be explained by the covariates using our model (Figure 5 ).

\section{Discussion}

Normally, an underlying infection is expressed as symptoms. Traditional approaches (qualitative and quantitative) for detecting co-infections are not always effective due to overlapping symptoms. As a result, clinicians prefer empirical antibiotic therapy with an emphasis on etiolo- gical staphylococci and streptococcal agents [14], [15]. Although the danger posed by bacterial co-infections in COVID-19 patients is recognized, the extent of co-infection with $S$. aureus has hitherto not been systematically evaluated. This meta-analysis found that overall, in the included studies, more than one-fourth of COVID-19 hospitalized patients had a co-infection (bacterial, fungal or viral), underscoring the need for establishment of protocols for the detection of coinfection to improve clinical data and patient therapy. Similarly, in about onefourth of recorded co-infections, S. aureus was the prevalent co-pathogen. This finding is consistent with a coinfection rate of 25\% (S .aureus) previously reported by [16]. The finding that MRSA was associated with over half of patients hospitalized with COVID-19/S. aureus coinfection is consistent with the prevalence rate of $50 \%$ reported by [16] in the 2009 influenza pandemic. The rates observed may be attributable to widespread antibiotic use on skin and nasopharyngeal microbiota, which may degrade the respective ecosystem [17]. The reported MRSA rates may be linked to non-judicious administration of broad-spectrum antibiotics to a large proportion of patients. The sensitivity of $S$. aureus culture methods could have been limited by the excessive use of antibiotics, so that our findings may have underestimated the actual situation.

In our meta-analysis, significant heterogeneity exists, which led us to an enquiry into its origin. The maximum likelihood model explained that covariates such as study type, study quality and country of study were associated with heterogeneity. The unexplained heterogeneity (20.48\%) may be due to differences between studies in 

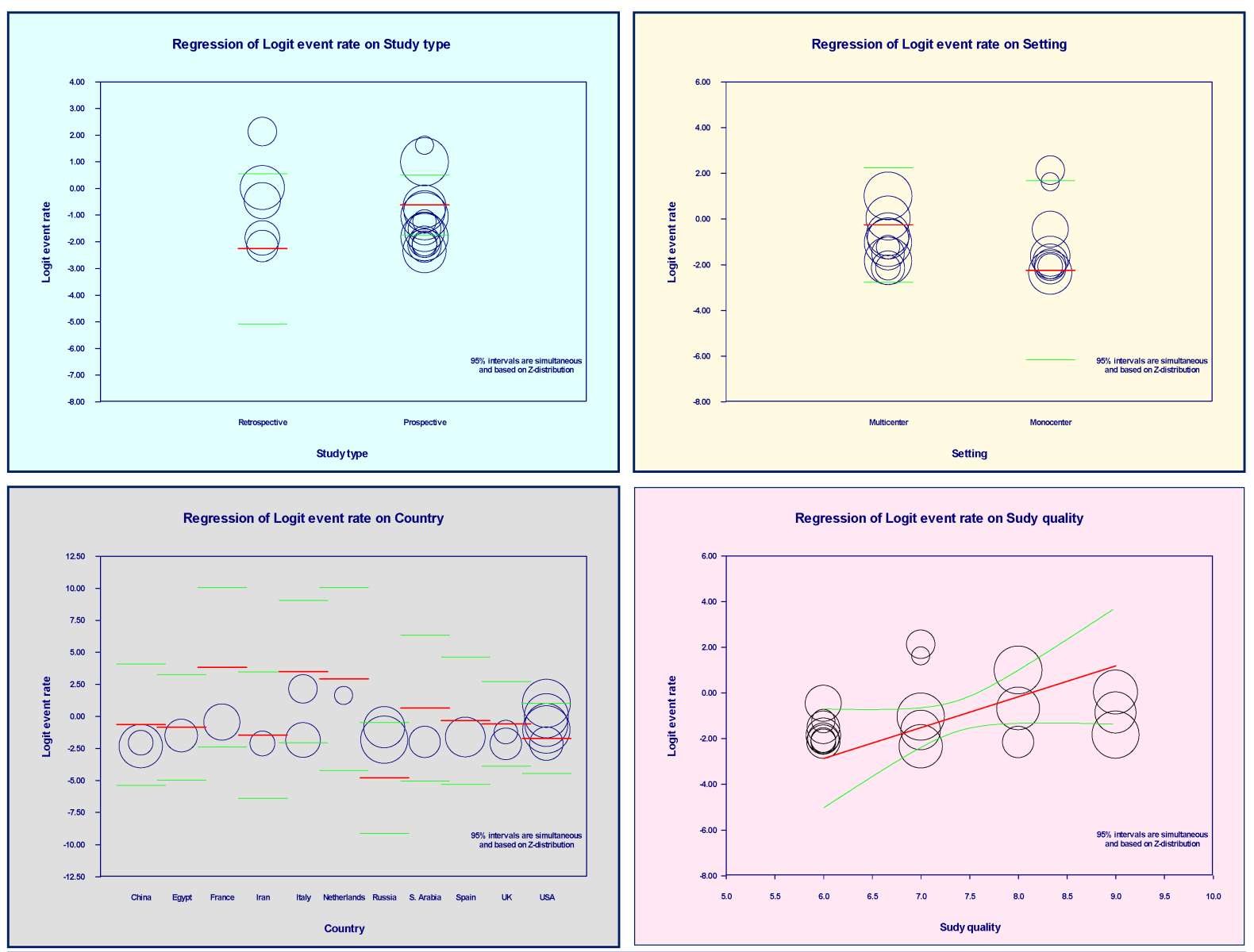

$R^{2}$ for Model 1, Random effects (ML), Z-Distribution, Logit event rate

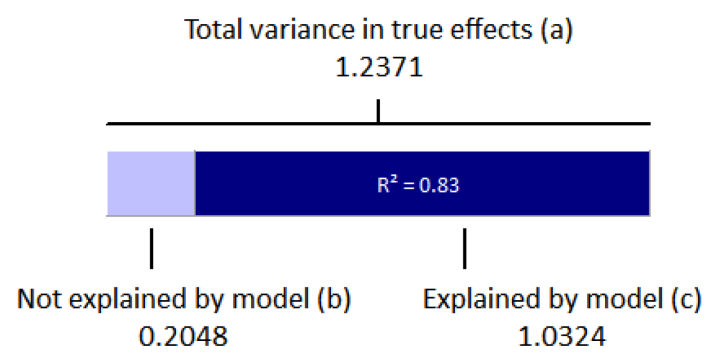

$$
R^{2}=\frac{\text { Explained (c) }}{\text { Total (a) }}=\frac{1.0324}{1.2371}=0.83
$$

Figure 5: Meta-regression of $S$. aureus co-infection effects and covariates (study type, study quality, study setting, country and combination of covariates)

terms of disease severity, patient co-morbidities, treatment differences, use of antibiotics prior to and during hospitalization, or other unidentifed covariates.

The strengths of the present study include our use of statistical models to assess the sources of heterogeneity, a systematic search strategy to classify potentially suitable studies from different sources, as well as scrutinizing the supplementary information of preprints and publications up to our search date. While research is ongoing, there are few studies documenting S. aureus and MRSA microbiological cultures among COVID-19 positive patients to date. This influenced the distribution of the studies covered. It is also likely that, considering the extraordinary conditions and immense burden on healthcare systems, patients with a suspected secondary infection would not have had extensive microbiological examinations. The data provided by the included studies did not distinguish between the sources of secondary infections and colonizers. This research focused solely on patients who were hospitalized and did not take into account patients who had not been hospitalized.

\section{Conclusion}

The pooled rates of S. aureus among COVID-19 patients documented in this study justify the concern of clinicians about the presence of $S$. aureus in co-infections. This data is not sufficient to support widespread- and often inappropriate empirical use of antibiotics in patients 
hospitalized with COVID-19, as reports of co-infection in admitted patients are scanty. Improved antibiotic stewardship can be accomplished through rapid diagnosis by longitudinal sampling of patients to allow targeted antimicrobial therapy.

\section{Notes}

\section{Competing interests}

The authors declare that they have no competing interests.

\section{Acknowledgements}

We thank Halima Salihu of the Department of Fisheries and Aquaculture, Usmanu Danfodiyo University Sokoto, Nigeria for her supportive remarks.

\section{Funding}

None was received.

\section{References}

1. Lansbury L, Lim B, Baskaran V, Lim WS. Co-infections in people with COVID-19: a systematic review and meta-analysis. J Infect. 2020 Aug;81(2):266-75. DOI: 10.1016/j.jinf.2020.05.046

2. Sharifipour E, Shams S, Esmkhani M, Khodadadi J, FotouhiArdakani R, Koohpaei A, Doosti Z, Ej Golzari S. Evaluation of bacterial co-infections of the respiratory tract in COVID-19 patients admitted to ICU. BMC Infect Dis. 2020 Sep;20(1):646. DOI: 10.1186/s12879-020-05374-z

3. Kluytmans J, van Belkum A, Verbrugh $\mathrm{H}$. Nasal carriage of Staphylococcus aureus: epidemiology, underlying mechanisms, and associated risks. Clin Microbiol Rev. 1997 Jul;10(3):50520. DOI: 10.1128/CMR.10.3.505-520.1997

4. Adeiza S, Onaolapo J, Olayinka B. Prevalence, risk-factors, and antimicrobial susceptibility profile of methicillin-resistant Staphylococcus aureus (MRSA) obtained from nares of patients and staff of Sokoto state-owned hospitals in Nigeria. GMS Hyg Infect Control 2020;15:Doc25. DOI: 10.3205/dgkh000360.

5. Alexander A, Vishwanath S, Sellvaraj A, Varma M, Saravu K, Chawla K. Methicillin-resistant Staphylococcus aureus nasal colonization in human immunodeficiency virus-infected patients. Annals of Tropical Medicine and Public Health. 2017;10(6):1809 13. DOI: $10.4103 /$ atmph.atmph_794_16

6. Duployez C, Le Guern R, Tinez C, Lejeune AL, Robriquet L, Six S, Loïez C, Wallet F. Panton-Valentine Leukocidin-Secreting Staphylococcus aureus Pneumonia Complicating COVID-19. Emerg Infect Dis. 2020 Aug;26(8):1939-41. DOI: 10.3201/eid2608.201413

7. Vaillancourt M, Jorth P. The Unrecognized Threat of Secondary Bacterial Infections with COVID-19. mBio. 2020 Aug;11(4). DOI: 10.1128/mBio.01806-20

8. WHO. Clinical management of severe acute respiratory infection (SARI) when COVID-19 disease is suspected. Interim guidance. Pediatr Med Rodz. 2020;16(1):9-26. DOI: 10.15557/PiMR.2020.0003
9. Hughes S, Troise O, Donaldson H, Mughal N, Moore LSP. Bacterial and fungal coinfection among hospitalized patients with COVID19: a retrospective cohort study in a UK secondary-care setting. Clin Microbiol Infect. 2020 Oct;26(10):1395-99. DOI: 10.1016/j.cmi.2020.06.025

10. Zhu X, Ge Y, Wu T, Zhao K, Chen Y, Wu B, Zhu F, Zhu B, Cui L. Co-infection with respiratory pathogens among COVID-2019 cases. Virus Res. 2020 Aug;285:198005. DOI: 10.1016/j.virusres.2020.198005

11. Santos WMD, Secoli SR, Püschel VAA. The Joanna Briggs Institute approach for systematic reviews. Rev Lat Am Enfermagem. 2018 Nov 14;26:e3074. DOI: 10.1590/1518-8345.2885.3074

12. Moher D, Shamseer L, Clarke M, Ghersi D, Liberati A, Petticrew M, Shekelle P, Stewart LA; PRISMA-P Group. Preferred reporting items for systematic review and meta-analysis protocols (PRISMAP) 2015 statement. Syst Rev. 2015 Jan;4. DOI: 10.1186/20464053-4-1

13. Adeiza SS. Nasal Colonization as a Risk Factor for Staphylococcal Infection: a Systematic Review and Meta-Analysis. SSRN Electronic Journal. 2018. DOI: 10.2139/ssrn.3658717

14. Zhang J, Zhou L, Yang Y, Peng W, Wang W, Chen X. Therapeutic and triage strategies for 2019 novel coronavirus disease in fever clinics. Lancet Respir Med. 2020 Mar;8(3):e11-e12. DOI: 10.1016/S2213-2600(20)30071-0

15. Buehler PK, Zinkernagel AS, Hofmaenner DA, Wendel Garcia PD, Acevedo CT, Gomez-Mejia A, Shambat SM, Andreoni F, Maibach M, Bartussek J, Hilty M, Frey PM, Schuepbach RA, Brugger SD. Bacterial pulmonary superinfections are associated with unfavourable outcomes in critically ill COVID-19 patients [Preprint]. medRxiv. 2020. DOI: 10.1101/2020.09.10.20191882

16. Klein EY, Monteforte B, Gupta A, Jiang W, May L, Hsieh YH, Dugas A. The frequency of influenza and bacterial coinfection: a systematic review and meta-analysis. Influenza Other Respir Viruses. 2016 Sep;10(5):394-403. DOI: 10.1111/irv.12398

17. Bogaert D, van Belkum A, Sluijter M, Luijendijk A, de Groot R, Rümke HC, Verbrugh HA, Hermans PW. Colonisation by Streptococcus pneumoniae and Staphylococcus aureus in healthy children. Lancet. 2004 Jun;363(9424):1871-2. DOI: 10.1016/S0140-6736(04)16357-5

18. Shah NS, Greenberg JA, McNulty MC, Gregg KS, Riddell J 4th, Mangino JE, Weber DM, Hebert CL, Marzec NS, Barron MA, Chaparro-Rojas F, Restrepo A, Hemmige V, Prasidthrathsint K, Cobb S, Herwaldt L, Raabe V, Cannavino CR, Hines AG, Bares SH, Antiporta PB, Scardina T, Patel U, Reid G, Mohazabnia P, Kachhdiya S, Le BM, Park CJ, Ostrowsky B, Robicsek A, Smith BA, Schied J, Bhatti MM, Mayer S, Sikka M, Murphy-Aguilu I, Patwari P, Abeles SR, Torriani FJ, Abbas Z, Toya S, Doktor K, Chakrabarti A, Doblecki-Lewis S, Looney DJ, David MZ. Bacterial and viral co-infections complicating severe influenza: Incidence and impact among 507 U.S. patients, 2013-14. J Clin Virol. 2016 Jul;80:12-9. DOI: 10.1016/j.jcv.2016.04.008

19. Crotty MP, Akins RL, Nguyen AT, Slika R, Rahmanzadeh K, Wilson $\mathrm{MH}$, Dominguez EA. Investigation of subsequent and co-infections associated with SARS-CoV-2 (COVID-19) in hospitalized patients [Preprint]. medRxiv. 2020. DOI: 10.1101/2020.05.29.20117176

20. Baunoch D, Wolfe A, Wang D, Gnewuch R, Zhao X, Halverson T, Cacdac P, Huang S, Lauterbach T, Luke N. Co-occurrence of SARS-CoV-2 and Respiratory Pathogens in the Frail Elderly [Preprint]. medRxiv. 2020. DOI: 10.1101/2020.06.24.20138941

21. Sharov KS. SARS-CoV-2-related pneumonia cases in pneumonia picture in Russia in March-May 2020: Secondary bacterial pneumonia and viral co-infections. J Glob Health. 2020 Dec;10(2):020504. 
22. Maes M, Higginson E, Pereira Dias J, Curran MD, Parmar S, Khokhar F, Cuchet-Lourenço D, Lux J, Sharma-Hajela S, Ravenhill B, Mahroof R, Solderholm A, Forrest S, Sridhar S, Brown NM, Baker S, Navapurkar V, Dougan G, Bartholdson Scott J, Conway Morris A. Secondary pneumonia in critically ill ventilated patients with COVID-19 [Preprint]. medRxiv. 2020. DOI: 10.1101/2020.06.26.20139873

23. Ramadan HK, Mahmoud MA, Aburahma MZ, Elkhawaga AA, EIMokhtar MA, Sayed IM, Hosni A, Hassany SM, Medhat MA. Predictors of Severity and Co-Infection Resistance Profile in COVID-19 Patients: First Report from Upper Egypt. Infect Drug Resist. 2020;13:3409-22. DOI: 10.2147/IDR.S272605

24. Nieuwenhuis MB, Van Biesen S, Juffermans NP. Response to "Co-infections in COVID-19 critically ill and antibiotic management: a prospective cohort analysis". Crit Care. 2020 Sep;24(1):591. DOI: 10.1186/s13054-020-03308-4

25. Nori P, Cowman K, Chen V, Bartash R, Szymczak W, Madaline T, Punjabi Katiyar C, Jain R, Aldrich M, Weston G, Gialanella P, Corpuz M, Gendlina I, Guo Y. Bacterial and fungal coinfections in COVID-19 patients hospitalized during the New York City pandemic surge. Infect Control Hosp Epidemiol. 2020 Jul 24;15. DOI: $10.1017 /$ ice. 2020.368

26. Song W, Jia X, Zhang X, Ling Y, Yi Z. Co-infection in COVID-19, a cohort study. J Infect. 2020 Oct 8. pii:S0163-4453(20)306484. DOI: 10.1016/j.jinf.2020.10.006

27. Punjabi CD, Madaline T, Gendlina I, Chen V, Nori P, Pirofski LA. Prevalence of methicillin-resistant (MRSA) in respiratory cultures and diagnostic performance of the MRSA nasal polymerase chain reaction (PCR) in patients hospitalized with coronavirus disease 2019 (COVID-19) pneumonia. Infect Control Hosp Epidemiol. 2020 Aug 26:1-2. DOI: 10.1017/ice.2020.440

28. Garcia-Vidal C, Sanjuan G, Moreno-García E, Puerta-Alcalde P, Garcia-Pouton N, Chumbita M, Fernandez-Pittol M, Pitart C, Inciarte A, Bodro M, Morata L, Ambrosioni J, Grafia I, Meira F, Macaya I, Cardozo C, Casals C, Tellez A, Castro P, Marco F, García F, Mensa J, Martínez JA, Soriano A; COVID-19 Researchers Group. Incidence of co-infections and superinfections in hospitalized patients with COVID-19: a retrospective cohort study. Clin Microbiol Infect. 2020 Jul 31:pii:S1198-743X(20)30450-X. DOI: 10.1016/j.cmi.2020.07.041

29. Alosaimi B, Hamed M, Alkadi H, Naeem A, Alanazi T, Alrehily S, Almutairi A, Zafar A. Influenza co-infection associated with severity and mortality in COVID-19 patients [Preprint]. Authorea. Sept 11 2020. DOI: 10.22541/au.159985934.49280104
30. Giacobbe DR, Battaglini D, Ball L, Brunetti I, Bruzzone B, Codda G, Crea F, De Maria A, Dentone C, Di Biagio A, Icardi G, Magnasco L, Marchese A, Mikulska M, Orsi A, Patroniti N, Robba C, Signori A, Taramasso L, Vena A, Pelosi P, Bassetti M. Bloodstream infections in critically ill patients with COVID-19. Eur J Clin Invest. 2020 Oct;50(10):e13319. DOI: 10.1111/eci.13319

31. Contou D, Claudinon A, Pajot O, Micaëlo M, Longuet Flandre P, Dubert M, Cally R, Logre E, Fraissé M, Mentec H, Plantefève G. Bacterial and viral co-infections in patients with severe SARSCoV-2 pneumonia admitted to a French ICU. Ann Intensive Care. 2020 Sep;10(1):119. DOI: 10.1186/s13613-020-00736-x

32. Aleman VD, Carbajal JAG, Velazquez FAA, Mohiuddin M, Varon $D$, Surani S, Varon J. Incidence of coinfection in patients with COVID-19. Chest. 2020;158(4)Suppl. DOI:10.1016/j.chest.2020.08.340

33. Calcagno A, Ghisetti V, Burdino E, Trunfio M, Allice T, Boglione L, Bonora S, Di Perri G. Coinfection with other respiratory pathogens in COVID-19 patients. Clin Microbiol Infect. 2020 Aug 19:pii:S1198-743X(20)30494-8. DOI: 10.1016/j.cmi.2020.08.012

\section{Corresponding author:}

\section{Suleiman Shuaibu Adeiza}

Department of Pharmaceutical Microbiology, Faculty of Pharmaceutical sciences, Ahmadu Bello University, Zaria, Kaduna, Nigeria, Phone: +23 48162208444 suleykestler2@gmail.com

\section{Please cite as}

Adeiza SS, Shuaibu AB, Shuaibu GM. Random effects meta-analysis of COVID-19/S. aureus partnership in co-infection. GMS Hyg Infect Control. 2020;15:Doc29.

DOI: 10.3205/dgkh000364, URN: urn:nbn:de:0183-dgkh0003647

\section{This article is freely available from}

https://www.egms.de/en/journals/dgkh/2020-15/dgkh000364.shtml

Published: 2020-11-27

\section{Copyright}

(C)2020 Adeiza et al. This is an Open Access article distributed under the terms of the Creative Commons Attribution 4.0 License. See license information at http://creativecommons.org/licenses/by/4.0/. 